\title{
Infection Rate and Outcomes of Watchman Devices: Results from a Single Center 14-Year Experience
}

\author{
Robert Ward ${ }^{1}$, Trevon McGill ${ }^{2}$, Fadi Adel $^{2}$, Shiva Ponamgi ${ }^{3}$, Samuel Asirvatham ${ }^{1}$, Larry \\ Baddour $^{1}$, David Holmes ${ }^{1}$, Daniel DeSimone ${ }^{1}$, and Christopher DeSimone ${ }^{1}$ \\ ${ }^{1}$ Mayo Clinic \\ ${ }^{2}$ Mayo Clinic Rochester \\ ${ }^{3}$ Mayo Clinic Health System - Albert Lea and Austin
}

October 1, 2020

\begin{abstract}
Background: Left atrial appendage occlusion with the Watchman device is an alternative strategy for stroke risk reduction in patients with non-valvular atrial fibrillation. There are rare case reports of Watchman associated infection. Currently, there is no formal study that evaluated the incidence and outcomes of Watchman-related infections. Methods: All patients who underwent Watchman implantation over a 14 year study period (July 2004 through December 2018) comprised our cohort. Baseline characteristics, procedural data, and post-implantation events were identified. Primary study outcomes included Watchman related infection, other cardiovascular device related infection, bacteremia, and mortality. Results: A total of 181 patients with an average age of 75, and a median CHA2DS2-VASc Score of 4 (interquartile range 2) and a median HAS-BLED Score of 3 (interquartile range 1), were included for analysis. A total of 534.7 patient years of follow up was accrued with an average of 2.9 years per patient. The most common indications for implantation included gastrointestinal bleeding (56 patients; $30.9 \%$ ) and intracerebral bleeding (51 patients; $28.2 \%$ ). During follow up, 38 patients (21\%) died. Six developed evidence of bacteremia. Only one developed an implantable cardioverter defibrillator (ICD) infection that required complete system extraction. None of the cohort developed Watchman-related device infection during the study period. Conclusion: In a single center study spanning a 14 year period, we report no Watchman-related devices infections. This is despite the presence of patients with bacteremia, as well as an ICD infection requiring extraction. These data suggest that Watchman devices are extremely unlikely to become infected.
\end{abstract}

Infection Rate and Outcomes of Watchman Devices: Results from a Single Center 14-Year Experience

Robert C. Ward, MD ${ }^{1}$, Trevon McGill, $\mathrm{MD}^{2}$, Fadi Adel, $\mathrm{MD}^{2}$, Shiva Ponamgi, MD ${ }^{3}$, Samuel J. Asirvatham, $\mathrm{MD}^{1}$, Larry M. Baddour, MD ${ }^{1,4}$, David R. Holmes Jr, MD ${ }^{1}$, Daniel C. DeSimone, $\mathrm{MD}^{1,4}$, Christopher V. DeSimone, $\mathrm{MD}, \mathrm{PhD}^{1}$

Author affiliations:

1. Department of Cardiovascular Disease, Mayo Clinic College of Medicine and Science, 200 First Street SW, Rochester, MN 55905, USA

2. Department of Medicine, Mayo Clinic, 200 First Street SW, Rochester, MN 55905, USA

3. Division of Cardiology, Creighton University, Omaha, NE 68124, USA

4. Division of Infectious Diseases, Department of Medicine, Mayo Clinic, 200 First Street SW, Rochester, MN 55905, USA 
Number of tables: 1

Total Word Count: 1,157

Corresponding author:

Christopher V. DeSimone, MD, PhD

Assistant Professor of Medicine

Division of Cardiovascular Diseases

Mayo Clinic, Rochester, MN 55905

Desimone.christopher@mayo.edu

\section{Introduction}

Left atrial appendage occlusion (LAAO) with a Watchman device (WD) (Boston Scientific, St. Paul, MN) is an alternative strategy to oral anticoagulation for embolic stroke risk reduction in patients with non-valvular atrial fibrillation $(\mathrm{AF})$.

There are rare case reports of WD- associated infection [1-3]. While the original Watchman trials reported on adverse outcomes broadly, they did not directly report on the incidence of WD-related infections or outcomes in the setting of blood-stream infections (BSI) [4-6].

We sought to evaluate the incidence, risk factors, and outcomes for WD-associated infections from a singlecenter cohort over a 14 year period.

\section{Methods}

This study protocol was approved by the Mayo Clinic IRB. A retrospective review of all patients who underwent WD implant from July 2004 through December 2018 was conducted. Patients had Intra-procedural trans-esophageal echocardiogram (TEE), 6 month, and 1 year TEE (with in person visits). If a device leak was noted $(>5 \mathrm{~mm})$, a 6 month return TEE was performed. After one year post-implant, patients were followed clinically.

From this cohort of patients, we performed detailed medical chart review to identify medical co-morbidities, indication for implantation, clinical and laboratory evidence of WD infection, BSI, and mortality data. A BSI was defined by at least one positive blood culture correlating to a clinical syndrome of infection. Valvular and WD-related endocarditis was defined by modified Duke Criteria[7].

Categorical variables are reported as percentages, and continuous variables are reported as mean $+/$ - standard deviation.

\section{Results}

A total of 181 patients underwent WD implantation at Mayo Clinic in Rochester, MN during the study period. Average age at implant was 75 years $( \pm 7.9)$. Patients were followed for a total of 534.7 patient years with an average follow-up time of 2.9 years. The median $\mathrm{CHA}_{2} \mathrm{DS}_{2}$-VASc Score was 4 (interquartile range 2) and the median HAS-BLED Score was 3 (interquartile range 1). Gastrointestinal bleeding ( $\mathrm{n}=56$, $30.7 \%)$, intracerebral hemorrhage $(\mathrm{n}=51,28.0 \%)$, and patient preference to avoid anticoagulation $(\mathrm{n}=23$, $12.6 \%$ ) constituted the most common indications for WD implantation.

There were no instances of WD-related infection or endocarditis throughout the follow up period. There were 6 patients who had evidence of bacteremia post implantation. Pathogens identified included viridans group Streptococcus, Escherichia coli, Streptococcus agalactiae, Micrococcusluteus, methicillin-susceptible Staphylococcusaureus, and Pseudomonas aeruginosa. Infectious syndromes included an implantable cardioverter defibrillator associated endocarditis, sepsis secondary to a urinary source, and sepsis secondary to a pulmonary source [Table 1] . Five of six BSI episodes occurred $>3$ months post implantation; one occurred 
26 days post implantation. None of the 6 patients who had BSI developed significant peri-device leak, defined as greater than $5 \mathrm{~mm}$ in size. No subsequent TEE showed evidence of device vegetation. A total of 37 patients died during follow-up with an overall mortality of $20.4 \%$, however no death was infection related.

\section{Discussion:}

This is the first systematic evaluation of a single cohort over a 14-year study period to report on WD infection or associated endocarditis, and no cases of WD-related infections were identified. Despite a small subset of patients developing BSI, there was no evidence of WD infection and no WD removed in an attempted cure of infection. There was one patient who required ICD extraction with subsequent BSI clearance. These findings suggest that WD infections are uncommon.

Complete endothelialization of the surface of cardiovascular devices reduces the risk of subsequent devicerelated infection and is thought to develop within three months of device implantation[8]. In theory, a WD with peri-device leak, and therefore more turbulent flow surrounding it, might be more susceptible to complicating device-related infection. None of the six patients in our cohort who had BSI had evidence of peri-device leak during follow-up. Only one of them developed BSI within 3 months of implantation.

One patient in our cohort developed an ICD infection due to viridans group Streptococcus . The patient underwent device extraction and a prolonged course of antibiotics with clearance of BSI. Despite sustained BSI due to ICD infection, the WD never developed infection.

No device-related infection was described in the original Watchman trials [4-6]. It is concerning, however whether monitoring for this complication was done in these trials; for example, one patient [1] in the PROTECT AF trial actually developed WD-related endocarditis that was not described in the original trial results.

\section{Conclusion:}

These findings support the low risk of device-related infections, even in the setting of BSI. Additional evaluation with specific follow-up for WD infection is warranted to further define the infectious proclivity of the WD.

\section{Table 1. Details of bloodstream infection cases}

\begin{tabular}{llll}
\hline Microbiological Isolate & Infectious source/syndrome & Days post implant & Device leak \\
\hline Viridans group Streptococcus & Infected ICD and IE & 1,897 & No \\
Escherichia coli & Urinary tract infection & 1,245 & No \\
Streptococcus agalactiae & No source identified & 1,085 & No \\
Micrococcus luteus & No source identified & 1,203 & No \\
Methicillin-susceptible Staphylococcus aureus & Pneumonia & 226 & No \\
Pseudomonas aeruginosa & Urinary tract infection & 26 & No \\
\hline
\end{tabular}

\section{References:}

1. Khumri, T.M., J.B. Thibodeau, and M.L. Main, Transesophageal echocardiographic diagnosis of left atrial appendage occluder device infection. Eur J Echocardiogr, 2008. 9 (4): p. 565-6.

2. Jensen, J., et al., Transesophageal Echocardiography to Diagnose Watchman Device Infection. CASE (Phila), 2020. 4 (3): p. 189-194.

3. Boukobza, M., et al., Convexity Subarachnoid Hemorrhage, Pseudomonas Aeruginosa (PA) Infective Endocarditis and Left Atrial Appendage Occluder (LAAO) Device Infection. A Case Report. Open Neuroimag J, 2017. 11 : p. 26-31.

4. Boersma, L.V., et al., Efficacy and safety of left atrial appendage closure with WATCHMAN in patients 
with or without contraindication to oral anticoagulation: 1-Year follow-up outcome data of the EWOLUTION trial. Heart Rhythm, 2017. 14 (9): p. 1302-1308.

5. Osmancik, P., et al., Left Atrial Appendage Closure Versus Direct Oral Anticoagulants in High-Risk Patients With Atrial Fibrillation. J Am Coll Cardiol, 2020. 75 (25): p. 3122-3135.

6. Reddy, V.Y., et al., 5-Year Outcomes After Left Atrial Appendage Closure: From the PREVAIL and PROTECT AF Trials. J Am Coll Cardiol, 2017. 70 (24): p. 2964-2975.

7. Durack, D.T., A.S. Lukes, and D.K. Bright, New criteria for diagnosis of infective endocarditis: utilization of specific echocardiographic findings. Duke Endocarditis Service. Am J Med, 1994.96 (3): p. 200-9.

8. Schwartz, R.S., et al., Left atrial appendage obliteration: mechanisms of healing and intracardiac integration. JACC Cardiovasc Interv, 2010. 3 (8): p. 870-7. 